\title{
Rethinking 'secondary' metabolism: physiological roles for phenazine antibiotics
}

\author{
Alexa Price-Whelan ${ }^{1}$, Lars E P Dietrich ${ }^{2}$ \& Dianne K Newman ${ }^{2,3}$ \\ Microorganisms exist in the environment as multicellular communities that face the challenge of surviving under nutrient- \\ limited conditions. Chemical communication is an essential part of the way in which these populations coordinate \\ their behavior, and there has been an explosion of understanding in recent years regarding how this is accomplished. \\ Much less, however, is understood about the way these communities sustain their metabolism. Bacteria of the genus \\ Pseudomonas are ubiquitous, and are distinguished by their production of colorful secondary metabolites called \\ phenazines. In this article, we suggest that phenazines, which are produced under conditions of high cell density and \\ nutrient limitation, may be important for the persistence of pseudomonads in the environment.
}

\begin{abstract}
Historically, microbiologists and chemists alike have categorized as 'secondary metabolites' a broad class of molecules produced at late stages of microbial growth in laboratory cultures. This nomenclature is, admittedly, pejorative, implying that these molecules are somehow less important than others to the cell that produces them. In particular, the traditional view is that secondary metabolites (i) do not contribute to the growth or survival of the producer (ii) are highly sensitive to the conditions stimulating their production (for example, medium composition) (iii) often have complex structures and (iv) have production rates that are decoupled from the doubling time of the cells ${ }^{1,2}$. Together, these leitmotifs present a conundrum: why would an organism limited for nutrients begin excreting large amounts of complex organic molecules? One reasonable and commonly stated answer is that they function as antibiotics, and are produced in copious quantities at this stage of growth to protect the producer from competitors ${ }^{3}$. In recent years, however, the idea that 'secondary' metabolites might have other functions, ranging from controlling gene expression ${ }^{4}$ to supporting growth or iron acquisition in microbial communities ${ }^{5,6}$, has become increasingly compelling. This is due, in large part, to the recognition that microbes typically exist in nature in biofilm communities ${ }^{7}$ and/or in a metabolically quiescent state $^{8}$; because the 'rules of the game' for metabolism under these conditions are virtually unknown, a re-examination of the function of secondary metabolites is warranted.

To illustrate the idea that secondary metabolites have the potential to perform primary metabolic functions, we will focus this review on a class of compounds known as 'phenazines', which have been of great interest to pharmaceutical and clinical research groups for the last 50 years 9 .
\end{abstract}

Divisions of ${ }^{1}$ Biology and ${ }^{2}$ Geological and Planetary Sciences, and ${ }^{3}$ Howard Hughes Medical Institute, California Institute of Technology, Pasadena, California 91125, USA. Correspondence should be addressed to D.K.N. (dkn@gps.caltech.edu).

Published online 18 January 2006; corrected 7 March 2006 (details online); doi:10.1038/nchembio764
Phenazines are heterocyclic compounds that are produced naturally and substituted at different points around their rings by different bacterial species (Table 1). Small modifications of the core phenazine structure give rise to a full spectrum of colors, ranging from the deep red of 5-methyl-7-amino-1-carboxyphenazinium betaine (aeruginosin A, 1) to the lemon yellow of phenazine-1-carboxylic acid (PCA, 2), to the bright blue of 1-hydroxy-5-methylphenazine (pyocyanin, 3) (Fig. 1). The combination and variety of functional groups added also determine the redox potential and solubility of these compounds, thus affecting their biological activity ${ }^{9-11}$.

The antagonistic effects of almost all of these derivatives are usually attributed to one general characteristic: redox activity. The 2-hydroxyphenazine-1-carboxylic acid (2-OHPCA, 4) produced by Pseudomonas aureofaciens is thought to kill off competing fungi through the production of reactive oxygen species ${ }^{12}$. Many of the effects of pyocyanin (PYO) and PCA on a diversity of eukaryotic hosts as well as bacteria are thought to result from oxidative activity or the inactivation of proteins important in the oxidative stress response ${ }^{13,14}$. Regardless of whether they are acting as antibiotics in the soil, or virulence factors during infection, the redox transformations of phenazines strongly influence their physiological effects in other organisms. A more detailed understanding of phenazine metabolism in competing or host cells is emerging, as very recently, researchers have begun to recognize that small variations in the reactivity of phenazines can give rise to differences in their elicited response ${ }^{15}$.

Concomitant with the development of ideas about phenazine activity during competition and infection, Pseudomonas aeruginosa and other phenazine-excreting bacteria have become popular model organisms for the study of quorum sensing and biofilm formation, two of the most active areas of research in the field of microbiology ${ }^{16-18}$. Whereas pharmaceutical and clinical groups have been focused on the physiological effects of these compounds in nonproducing organisms, microbial physiologists and geneticists have typically viewed phenazines as metabolites that perform only secondary functions. As a result, despite research interest in both the bio- 


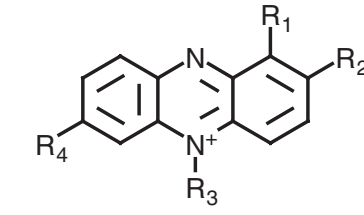

\begin{tabular}{|c|c|c|c|c|c|c|c|c|}
\hline No & Name & $\mathrm{R}_{1}$ & $\mathrm{R}_{2}$ & $\mathrm{R}_{3}$ & $\mathrm{R}_{4}$ & $K_{\text {ow }}$ ox & $K_{\text {ow red }}$ & $\mathrm{E}^{\mathrm{O}^{\prime}}(\mathrm{mV})$ \\
\hline 2 & Phenazine-1-carboxylic acid (PCA) & $\mathrm{COOH}$ & & & & 2.17 & 3.72 & -177 \\
\hline 3 & Pyocyanin (PYO) & $\mathrm{OH}$ & & $\mathrm{CH}_{3}$ & & 1.60 & 2.89 & -34 \\
\hline 4 & $\begin{array}{l}\text { 2-Hydroxyphenazine-1-carboxylic acid } \\
\text { (2-OHPCA) }\end{array}$ & $\mathrm{COOH}$ & $\mathrm{OH}$ & & & 2.54 & 3.32 & NA \\
\hline 6 & 1-Hydroxyphenazine (1-OHPHZ) & $\mathrm{OH}$ & & & & 1.81 & 2.35 & -172 \\
\hline
\end{tabular}

Table 1 Characteristics of some phenazines excreted by pseudomonads ${ }^{90-92}$. Octanol-water partition coefficients $\left(K_{\text {ow }}\right)$ were calculated using the KOWWIN demo program available at http://www.syrres.com/esc/est_kowdemo.htm. $E^{\circ \prime}$, standard redox potential, pH 7; NA, not available.

logical activity of the compounds themselves and in the physiology of their producers, the primary functions of phenazines for producing organisms such as the pseudomonads are still unknown. This is surprising, especially given that phenazine production and reduction is evident in many of the Pseudomonas cultures that microbiologists prepare for their work (Fig. 2) and that the mechanisms thought to be responsible for phenazine metabolism in non-producers (for example, reduction by NADH or glutathione, interaction with the respiratory chain) are present in most organisms ${ }^{19,20}$. That phenazines and other excreted compounds (i) react with common primary metabolites (ii) are potentially transformed by enzymes active in central metabolic pathways and (iii) induce gene expression calls into question their categorization as secondary metabolites. We will discuss here the recent discoveries that lead to new hypotheses about the relevance of phenazine metabolism in the context of the lifestyles of their producers.

\section{Occurrence of phenazine production}

Phenazines are first mentioned in the literature as early as the 1860 s, when French researchers and clinicians noticed a blue coloration in the pus and sputum, or respiratory secretion, of infected patients. Carle Gessard and others examined the pus microscopically and identified a rod-shaped bacterium residing in these wounds, and upon isolating the organism discovered that it was responsible for the bluish tint. For this trait, they named the species Bacillus pyocyaneus, and it has since been renamed Pseudomonas aeruginosa, for the Latin aerugo, which refers to the blue-green rust of copper ${ }^{21} . P$. aeruginosa is widespread in terrestrial habitats, can grow in both marine and freshwater environments, and is known for its ability to infect a diversity of hosts, ranging from plants to humans ${ }^{21-23}$. The $P$. aeruginosa laboratory strains PAO1 and PA14 are capable of producing at least four different phenazine derivatives ${ }^{24}$.

Several other Pseudomonas species are also phenazine producers and are known for their potential in biocontrol applications, in which an organism that inhibits the growth of crop pathogens is enriched in the soil to enhance crop yields. Representatives include the strains P. aureofaciens 30-84, P. fluorescens 2-79 and P. chlororaphis PCL1391. Along with $P$. aeruginosa, these isolates all produce one or more phenazines and differ in their biosynthetic capabilities with respect to phen- bacterial genera, including other proteobacteria, such as Brevibacterium, Burkholderia and Xanthomonas, as well as the Gram-positive genus Streptomyces and even the archaeal genus Methanosarcina ${ }^{22,27,28}$. This review will focus on the pseudomonad phenazines, because they are the best studied with respect to biosynthesis, but will also include a discussion of the methanophenazine produced by Methanosarcina mazei Gö1, because it is the only phenazine thus far that has been unequivocally shown to have an important role in catabolism ${ }^{27}$.

\section{Roles in eukaryotic physiology and pseudomonad persistence}

Phenazines in infection. Some of the most thorough studies that have been conducted to investigate the physiological consequences of phenazine exposure are those of Britigan and colleagues, who have reported the many effects of phenazines produced by $P$. aeruginosa during infection of the human lung ${ }^{29}$. Phenazine production plays an important role in both acute and chronic $P$. aeruginosa lung infections, which are frequent causes of mortality in patients who have cystic fibrosis or otherwise impaired lung function ${ }^{21}$. PYO has been detected in the sputum of patients with chronic $P$. aeruginosa infections at concentrations as high as $27 \mu \mathrm{g} \mathrm{ml}^{-1}$ (ref. 30), and the administration of purified PYO at comparable concentrations in laboratory mice has been shown to induce neutrophil influx in lung tissue ${ }^{31}$. Phenazine production is a common trait in strains of $P$. aeruginosa isolated from patients with cystic fibrosis $^{32}$, and mutant versions of the $P$. aeruginosa strains PAO1 and PA14 that are unable to synthesize PYO are attenuated in both acute and chronic mouse lung infection models ${ }^{33}$.

The oxidative activity of phenazines in particular has been shown to be important in pathogenesis during $P$. aeruginosa lung infection ${ }^{14}$. Both PYO and PCA can increase oxidant formation in human airway epithelial cells through a number of mechanisms including the oxidation of glutathione and NADH and inhibition of antioxidant enzymes ${ }^{13,15,34}$. Once it is reduced, PYO can then react with oxygen, forming superoxide radical and hydrogen peroxide ${ }^{35}$. Pyocyanin radical is also formed as an intermediate during its redox cycling, and can further contribute to the formation of reactive oxygen species ${ }^{36-39}$. These insults to the host cell's internal redox balance may lead to increased secretion and thereby contribute to the generation of sputum, the physical and nutritional substrate for $P$. aeruginosa in the lungs of individuals with cystic fibro$\operatorname{sis}^{40,41}$. The generation of radical species (of phenazines and oxygen) 
a

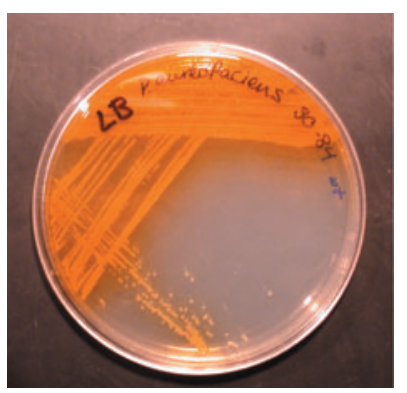

b

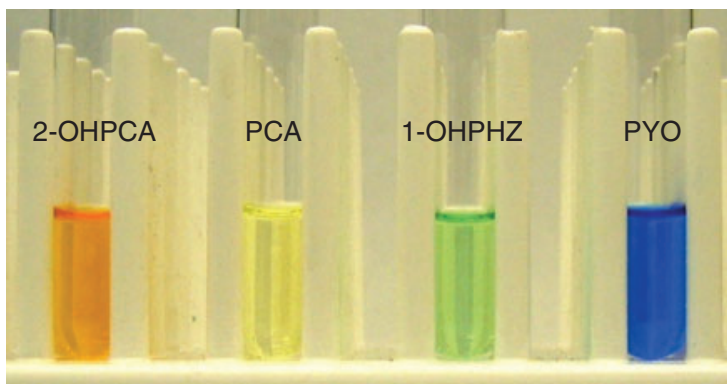

Figure 1 Phenazines are colorful, diffusible bacterial metabolites. (a) Streak plate of the biocontrol strain $P$. aureofaciens 30-84. The phenazine 2-OHPCA turns the agar bright orange. (b) Aqueous solutions of some of the phenazines produced by various Pseudomonas strains.
In Pseudomonas spp., the phenazine biosynthetic pathway branches off from the shikimic acid pathway, which is also the source for metabolites such as the aromatic amino acids, siderophores and quinones (Scheme 1) ${ }^{45-47}$. Genes encoding the phenazine biosynthetic enzymes are arranged in one core operon, phzABCDEFG, in most phenazineproducing pseudomonads ${ }^{25,48}$. Such an operon exists in the genome of $P$. aeruginosa in duplicate, and the expression of the two copies of the operon is differentially regulated $^{24}$. In many strains, additional genes involved in phenazine decoration, such as $p h z M, p h z S, p h z O$ and $p h z H$, are present in single copy and can be located proximally to the core operon or elsewhere in the genome ${ }^{26}$;

is potentially harmful to other microbes competing for resources in the lung, such as Staphylococcus aureus, and may help P. aeruginosa to persist in this environment ${ }^{42}$. However, recent work has demonstrated that phenazine production is beneficial to the growth or survival of $P$. aeruginosa in mouse infection models even in the absence of competing organisms, implying that these compounds may additionally provide a physiological benefit to their producers during infection ${ }^{33}$.

Phenazines in soil ecosystems. The other well-studied niche for phenazine-producing pseudomonads is the rhizosphere, the zone surrounding the roots of plants. As is the case for P. aeruginosa in an infected lung, species such as $P$. aureofaciens, $P$. fluorescens and P. chlororaphis compete in this ecosystem with other organisms for resources. More importantly, they compete for colonization sites on the roots of agriculturally important crops, where they thrive as microcolonies (biofilms) and protect the plants from pathogenic fungi. Phenazines are thought to be important in this competition, and consistent with this, phenazineproducing strains of $P$. aureofaciens and $P$. fluorescens are better able to colonize the roots of wheat plants and persist in the rhizosphere than are phenazine-lacking mutants ${ }^{43}$.

The toxicity of phenazines to bacteria and fungi typically present in the rhizosphere has been demonstrated, and again is thought to be mostly due to the generation of reactive oxygen species ${ }^{12}$. If biocontrol strains did use phenazine toxicity as a weapon to compete with indigenous soil populations for resources, one would expect the composition of rhizosphere communities to change after exposure to phenazines; however, this does not occur. The overall number of organisms competing with $P$. fluorescens for resources does not decline after this strain has colonized the $\operatorname{root}^{44}$. This implies that it is not just the antibiotic activity of phenazines that is important for the ability of their producers to compete in the soil. As is the case for phenazines produced by P. aeruginosa, there is evidence indicating that phenazines have roles in the physiology and thus the ecological competence of the biocontrol pseudomonads.

\section{Biosynthesis of phenazine derivatives}

As mentioned above, the early observation that phenazines are produced in stationary phase in typical lab cultures led to the general view that they were unimportant in metabolism. However, we now know that phenazines are produced in biofilms and as a result are present in detectable quantities in the rhizosphere and in the lungs of cystic fibrosis patients. This has fueled interest in the phenazine biosynthetic pathway and the environmental factors that influence expression of the biosynthetic genes. The complexity of the regulation of phenazine biosynthesis is only just beginning to be appreciated and is consistent with the high degree of biological activity shown by these compounds. currently, little is known about how these genes are regulated.

Expression of phenazine biosynthetic genes is regulated by multiple mechanisms, which are strongly influenced by environmental conditions. One of the primary factors governing phenazine production is population density, and in P. aeruginosa this dependency is effected by at least three quorum-sensing systems ${ }^{49,50}$. Bacteria participating in quorum sensing release intercellular signals such as $N$-acyl-L-homoserine lactones (AHLs, 7 and 8) and 2-heptyl-3-hydroxy-R-quinolone (the Pseudomonas quinolone signal, PQS, 9) into the environment, where they can be taken up by neighboring cells of the same or different species (Fig. 3). Inside the cell, these compounds induce the expression of genes for their own biosynthesis-as well as many other genes important in virulence, competition and behavior-when they accumulate to a threshold concentration ${ }^{18}$. The dependence of phenazine biosynthesis on cell density has also been demonstrated for biocontrol pseudomonads, and in these species is mediated by a seemingly less complex quorum-sensing network ${ }^{51-53}$.

In addition to cell density, numerous environmental factors have been identified that affect the regulation of phenazine biosynthesis, including

\section{a}

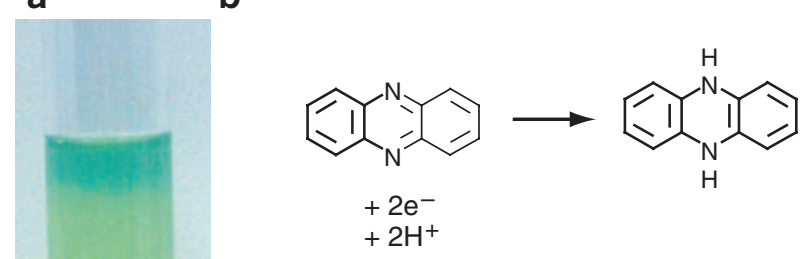

C

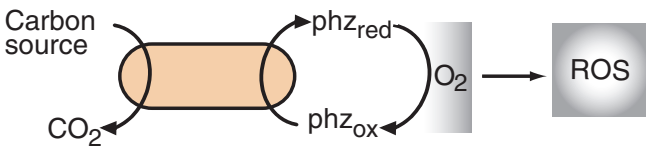

Figure 2 Pseudomonads stimulate phenazine reduction. (a) Characteristic gradient formed by standing cultures of $P$. aeruginosa. Bacterial respiration renders most of the culture anoxic. Phenazines (phz) are reduced and, in the case of PYO, become colorless. The darker blue at the top represents oxidized PYO. (b) Half-reaction representing generic two-electron phenazine reduction. (c) Schematic of phenazine reduction and auto-oxidation responsible for gradient formation in standing cultures. Reduced phenazines are oxidized abiotically by oxygen, generating reactive oxygen species (ROS). 


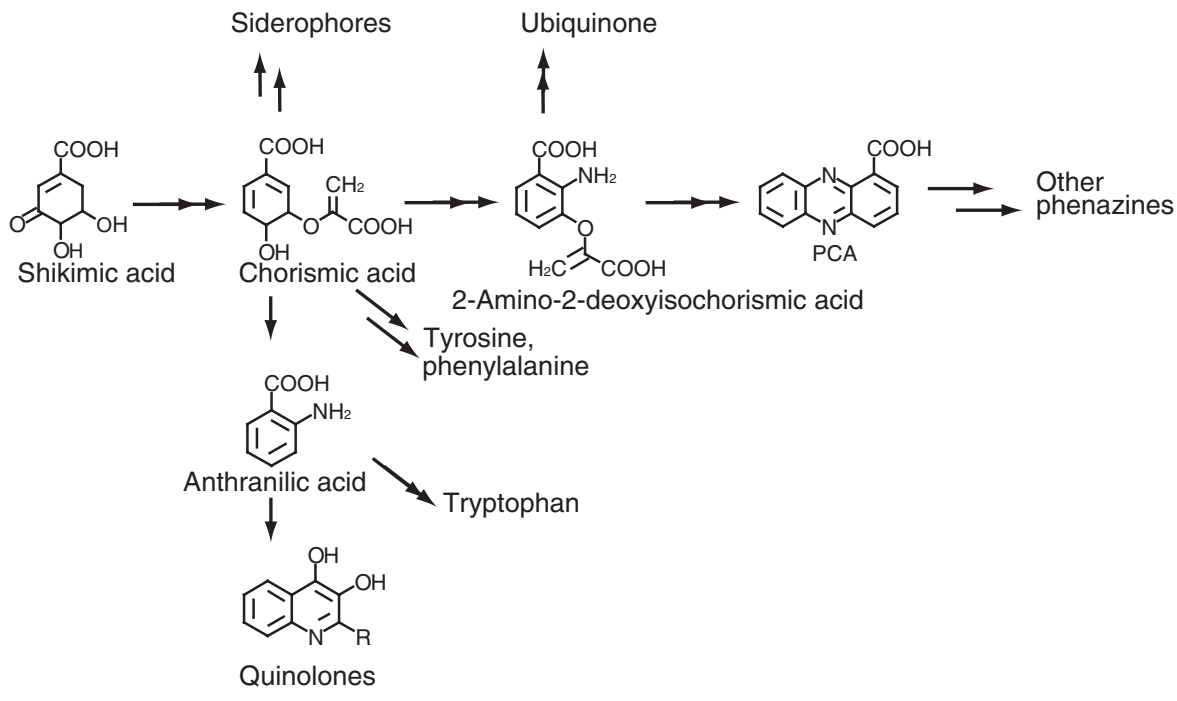

Scheme 1 Phenazine biosynthesis and its relation to the shikimic acid pathway in pseudomonads $24,45,46,93$.

oxygen, iron and phosphate concentration as well as the nature of the carbon sources available ${ }^{54}$. For many of these effects, it is not entirely clear whether they are above quorum sensing in the regulatory cascade (for example, iron limitation induces quorum-signal production, which in turn induces phenazine biosynthesis) or are the result of regulators acting independently of quorum sensing 55 . The GacA/S two-component system, which effects global changes in transcription, has been implicated in the control of phenazine biosynthesis and is thought to act by regulating quorum sensing, but there is also evidence that it affects phenazine gene expression through other mechanisms ${ }^{56-58}$. Repressors of phenazine biosynthesis have been identified in the plant symbionts $P$. aureofaciens and $P$. chlororaphis. Mutations in these repressors seem to override the quorum-sensing regulation of phenazine biosynthesis in these organisms, resulting in constitutive phenazine production ${ }^{58,59}$.

\section{Intercellular signaling: a regulatory role for phenazines}

Recent work from our laboratory has contributed to understanding of the complexity of the $P$. aeruginosa quorum-sensing system and the place of phenazines in this cell density-dependent cascade. We have found that, in addition to being regulated by cell-cell communication, phenazines themselves can act as intercellular signals. Our work indicates that PYO is the physiological inducer of a set of genes previously identified as members of the quorum-sensing regulon. PYO acts downstream of PQS, which previously had been deemed the terminal signal in the $P$. aeruginosa quorum-sensing cascade. PYO's function as a quorum signal explains what was thought to be a delayed response in the expression of a specific set of genes in response to PQS ${ }^{49}$. We now understand that these genes are expressed later than those induced directly by PQS because PQS first has to upregulate the biosynthesis of phenazines so that PYO can subsequently induce its stimulon (ref.60 and unpublished data) (Fig. 3).

The signaling function of PYO makes it the newest addition to the growing list of small molecules excreted by $P$. aeruginosa that have been shown to perform multiple functions ${ }^{61}$. PQS, like PYO, was also long recognized for its antibiotic and virulence properties before its role in signaling was elucidated ${ }^{62}$. Recent studies have demonstrated that certain AHL and quinolone derivatives chelate iron ${ }^{63,64}$, raising the possibility that these metabolites facilitate iron uptake in vivo. The accu- mulating knowledge about the chemistry and biological activity of small molecules excreted by Pseudomonas spp. calls for a re-evaluation of our categorizations of these compounds. Rather than clearly performing one dedicated purpose, they seem to be capable of multiple roles. To determine the most important physiological effects of phenazines and other small metabolites in the environment and during infection, we will need to understand the physiological conditions allowing, requiring and regulating their activities. Elucidating their mechanisms of action at the molecular level may also provide indications of the conditions relevant for activity.

\section{Other physiological roles}

In addition to inducing gene expression, phenazines also act as substrates in intracellular redox transformations. This further metabolism of phenazines, subsequent to their biosynthesis, can be observed as a color change in pseudomonad cultures that have become limited for terminal electron acceptors. This is because pseudomonads reduce their own phenazines, and changing the oxidation state of a phenazine changes its absorbance spectrum. In cultures of the bacterium P. aeruginosa, this is observed as a loss of blue coloration, because the main phenazine produced by this organism, PYO, changes from blue to colorless upon reduction at neutral $\mathrm{pH}$ (Fig. 2) ${ }^{65}$. This activity has also been demonstrated in oxygen-limited cultures of the bacterium $P$. chlororaphis, which can use its phenazine product, phenazine-1-carboxamide (PCN, 5) to reduce extracellular iron oxides ${ }^{5}$. Although a good deal of research effort has gone toward understanding phenazine reduction by mammalian cells, less work has been done to elucidate the mechanisms of the phenazine reduction that is readily observed in pseudomonad cultures. Here, we will discuss what is known about phenazine reduction and its physiological functions in phenazine-producing and non-producing prokaryotes.

Phenazines as 'respiratory pigments.' Based on the redox potentials, metabolism and solubilities of phenazines, it has been proposed that phenazines act as electron acceptors in cellular energy generation or in the maintenance of the intracellular redox balance ${ }^{66}$. Studies conducted by Ernst Friedheim in the 1930s, in which he observed that PYO increased the oxygen consumption of cell suspensions of P. aeruginos $a^{67}$, support this idea. Several reports on the interactions of PYO and 1-hydroxyphenazine (1-OHPHZ, 6) with the mammalian respiratory chain were published in the decades that followed. 1-OHPHZ, but not PYO, was shown to inhibit respiration at the level of ubiquinone in the electron transport chain of mammalian cells. It was concluded, based on measurements of oxygen depletion (which did not decrease in the presence of PYO) that PYO did not inhibit mammalian cell respiration ${ }^{19,68}$. We question this interpretation, however, given that PYO can accept electrons from $\mathrm{NADH}$ and transfer them to oxygen; accordingly, what was thought to be normal respiration may actually have been short-circuiting of the electron transport chain by PYO. In contrast, it makes sense that oxygen depletion was not observed in the presence of 1-OHPHZ given that reduced 1-OHPHZ does not react with oxygen at appreciable rates ${ }^{69}$.

Notably, the interactions of phenazines with the pseudomonad respiratory chain are largely unknown. Numerous groups have observed that both synthetic and natural phenazines are reduced by prokaryotes, but in most cases the physiological effect of this reduction has not been evaluated $^{70,71}$. One exception is the role of phenazine reduction in the 

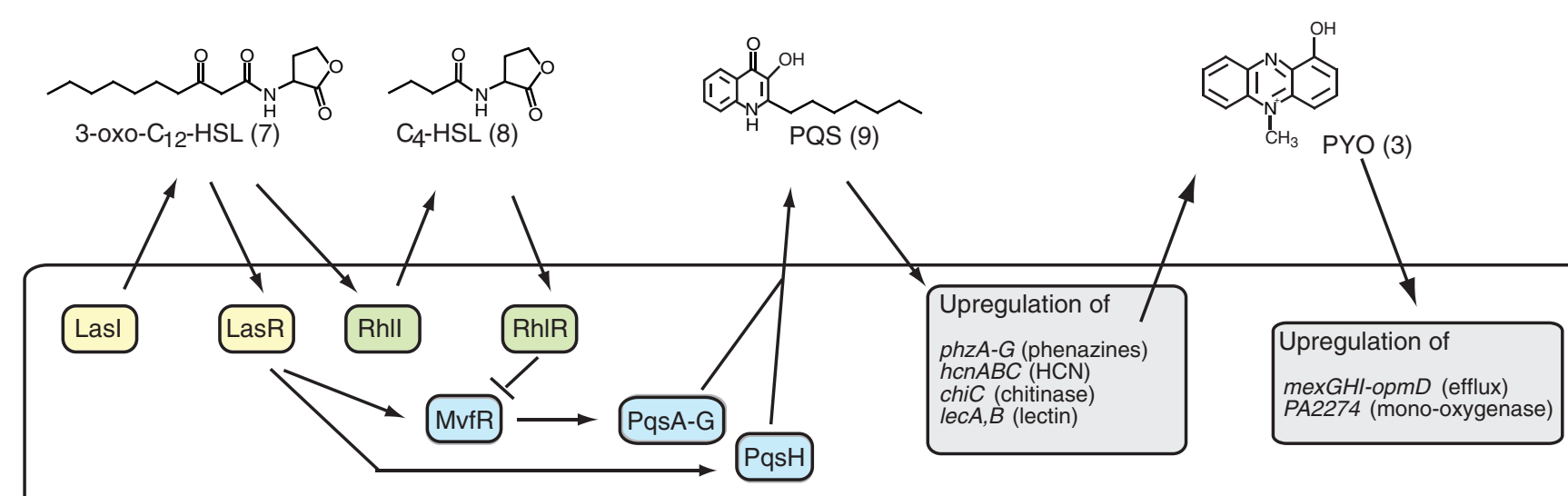

Figure 3 Model of the quorum-sensing network in Pseudomonas aeruginosa. The quorum-sensing network in Pseudomonas aeruginosa comprises a cascade of three types of signaling molecules that function in a growth stage-dependent manner. The AHLs 3-oxo- $\mathrm{C}_{12}-\mathrm{HSL}$ and $\mathrm{C}_{4}-\mathrm{HSL}$ are released in exponential phase and control the production of the quinolone PQS. PQS accumulates in late exponential phase and is required for the synthesis of phenazines. Recent findings from our laboratory show that the phenazine PYO upregulates genes that have previously been demonstrated to be controlled by quorum sensing, establishing PYO as a signaling molecule ${ }^{50,94}$.

respiratory chain of $M$. mazei Gö1 (ref. 72). This archaeon produces methanophenazine, a phenazine derivative with a pentaisoprenoid side chain, and can utilize phenazines in lieu of quinones in electron transport. In vitro, methanophenazine has been shown to accept electrons from hydrogen or a reduced coenzyme by means of the activity of either of two membrane-bound dehydrogenases, one of which is homologous to the NADH dehydrogenase found in bacteria and mitochondria. Reduced methanophenazine can then donate electrons to another cofactor in a reaction catalyzed by a membrane-bound heterodisulfide reductase $\mathrm{e}^{73}$. In vivo, these respiratory enzymes couple electron transport to the translocation of protons, generating a proton gradient that can

\section{Secondary metabolites}<smiles>Cn1c2cccc(=O)c-2nc2ccccc21</smiles>

C<smiles>CCCCCCCCCCCCCCCCC</smiles>

Primary metabolites<smiles>Cc1cc2nc3c(=O)[nH]c(=O)nc-3n(P)c2cc1C</smiles>

d

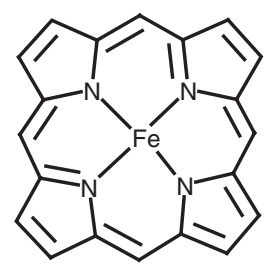

Figure 4 Key structural elements of secondary metabolites resemble those of cofactors that have critical roles in energy metabolism. (a) Pyocyanin. (b) Generic flavin. (c) Prodigiosin, an antibiotic produced by Serratia marcescens. (d) Generic heme ${ }^{95,96}$. be used to make $\mathrm{ATP}^{74}$. In M. mazei, therefore, phenazine reduction is not only crucial to energy metabolism in that it reoxidizes the NADH analog found in methanogens, but it is presumably also required for ATP synthesis ${ }^{72,75}$. Whether phenazine reduction can be similarly used by phenazine-producing pseudomonads to reoxidize $\mathrm{NADH}$ or generate ATP is still an open question.

Phenazines in redox homeostasis and iron acquisition Advances in our understanding of bacterial communities have provided an environmental context for the hypothesis that pseudomonads benefit by reducing phenazines. It has been proposed that the reduction of diffusible small molecules is advantageous during growth in a biofilm, a surface-attached population of bacteria suspended in an excreted matrix $^{66}$. The diffusion rate of oxygen through a biofilm is thought to be slow, and cells at the base of an aerobic biofilm become limited for oxidants ${ }^{76,77}$. Under this condition, phenazines could allow bacteria to generate energy for growth or help maintain redox homeostasis by acting as electron acceptors for the reoxidation of accumulating NADH. Indeed, maintaining a proper redox balance in the pyridine nucleotide pool is essential for metabolism ${ }^{78}$, and recent work from our lab indicates that $\mathrm{P}$. aeruginosa phenazine-negative mutants have higher intracellular $\mathrm{NADH} / \mathrm{NAD}^{+}$ratios in stationary phase than does the parent strain in planktonic cultures(unpublished data). This suggests that an important role for phenazines could be to serve as intracellular redox 'buffers'.

Various research groups have recently become more interested in phenazine reduction by biofilm-forming bacteria because phenazines make excellent electron transfer mediators to electrodes in biological fuel cells ${ }^{79}$. In biological fuel cells deployed in the environment as well as those set up in laboratories, bacteria often grow as biofilms attached to the electrode surface ${ }^{80,81}$. That phenazines facilitate electron transfer to electrodes has been demonstrated by Zeikus and colleagues, who investigated the ability of Escherichia coli to reduce the synthetic phenazine neutral red. They showed that E. coli is able to use this synthetic phenazine as an electron transfer mediator in the reduction of iron oxide, and presented evidence indicates that hydrogenase is at least partially responsible for this 
capability $^{71}$. Our group as well as others have shown that other synthetic dyes, with structures resembling those of phenazines, are reduced by Bacillus, Lactococcus and Shewanella species ${ }^{5,70,82}$. The Verstraete group has shown that phenazine production enhances power output from microbial fuel cells, and that biofuel cells enrich for phenazine-producing organisms; whether phenazine production influences the growth or survival of these bacteria in this context remains unclear ${ }^{83,84}$.

Aside from these proposed roles in energy generation, it has been suggested that phenazine reduction could act to make iron more available to the producing organism. PYO may assist infectious $P$. aeruginosa in the acquisition of iron by reducing it and freeing it from transferrin, a protein that normally sequesters iron so that it is available only to the human host ${ }^{85}$. As mentioned above, $P$. chlororaphis has been shown to reduce iron oxides through electron transfer to $\mathrm{PCN}$, and it is thought that this ability may be important in the rhizosphere, where iron is present predominantly in an insoluble form ${ }^{5}$. An examination of the relationship between iron availability and the regulation of phenazine biosynthesis, however, presents a complicated picture that neither refutes nor supports a role for these compounds in iron acquisition. Although in many cases it has been reported that phenazine production is enhanced in iron-deprived cultures, other studies have demonstrated a requirement for iron in media optimized for phenazine biosynthesis $54,85,86$. These differences probably arise from the high degree of variability with respect to other parameters, such as carbon source and the concentrations of oxygen and various salts. Perhaps the best way to ascertain whether or not iron availability has relevance to phenazine production will be to observe its effects under conditions that imitate the most common habitats for phenazine-producing pseudomonads ${ }^{41}$.

How are phenazines reduced? Although we are beginning to recognize the potential physiological importance of phenazine redox cycling, we have yet to identify the mechanisms by which pseudomonads catalyze the reduction of these compounds. On the basis of their low redox potentials and the mechanisms for phenazine reduction identified in eukaryotic cells, we might predict that NADH or glutathione would act as electron donors in these reactions. In the case of PYO, an enzyme may not be required, given that this compound reacts with these electron donors spontaneously. However, the relatively rapid reduction of other phenazines by bacterial suspensions is thought to require one or more enzymes ${ }^{71}$. The flavin-like structures of phenazines lead us to hypothesize that flavin-cofactored enzymes, capable of accepting electrons from lowpotential donors such as $\mathrm{NADH}$, might be responsible for catalyzing these transformations. If such an enzyme were associated with the respiratory chain, pseudomonads might be able to couple the reduction of their own excreted metabolites to the generation of a proton-motive force; in the absence of a better electron acceptor, they could utilize phenazines to generate ATP. Alternatively, if the enzyme were not associated with respiration, or were not a coupling site for proton transfer, phenazine reduction could still serve the valuable function of reoxidizing $\mathrm{NADH}$ under conditions in which the intracellular NADH/NAD ${ }^{+}$pool had shifted toward reducing.

\section{Conclusions}

As more is learned about the chemistry and biological activity of phenazines, we begin to question their categorization as 'secondary' metabolites. This compels us to rethink secondary metabolism as a phenomenon more generally. It is striking that the conditions under which secondary metabolites are produced in laboratory cultures (that is, stationary phase) are effectively the same as those that define many microbial habitats in nature ${ }^{8}$. Consistent with this, gene expression and physiological attributes seem to be very similar in stationary-phase planktonic cultures and biofilms ${ }^{87,88}$. As we have discussed for phenazines, stationary-phase metabolites can allow bacteria to sense the conditions of their surroundings and induce appropriate changes in gene expression; moreover, they can facilitate extracellular electron transfer to oxidants such as insoluble iron ${ }^{5,24}$ and have the potential to play a role in redox homeostasis. Notably, phenazines are only one class among the myriad natural products made by microorganism ${ }^{89}$, many of which bear intriguing structural resemblances to cofactors that have important roles in primary metabolism (Fig. 4). Now that we are beginning to understand stationary-phase physiology and its ecological relevance, it is time to revisit the roles of these compounds in gene expression and survival. We suspect that such studies will further blur the line between primary and secondary metabolism and will lead to a more complete picture of the mechanisms allowing organisms to persist in dynamic environments.

\section{ACKNOWLEDGMENTS}

The work was supported by an NIH training grant (A. P.-W.) and a postdoctoral EMBO fellowship (L.E.P.D.) administered by the California Institute of Technology, the Howard Hughes Medical Institute (D.K.N.) and the Packard Foundation.

\section{COMPETING INTERESTS STATEMENT}

The authors declare that they have no competing financial interests.

Published online at http:/www.nature.com/naturechemicalbiology/ Reprints and permissions information is available online at http://npg.nature.com/ reprintsandpermissions/

1. Madigan, M.T., Martinko, J.M. \& Parker, J. Brock Biology of Microorganisms (PrenticeHall, Upper Saddle River, NJ, USA, 2000).

2. Vining, L.C. Functions of secondary metabolites. Annu. Rev. Microbiol. 44, 395-427 (1990).

3. Firn, R.D. \& Jones, C.D. Natural products - a simple model to explain chemical diversity. Nat. Prod. Rep. 20, 382-391 (2003).

4. Goh, E.B. et al. Transcriptional modulation of bacterial gene expression by subinhibitory concnetrations of antibiotics. Proc. Natl. Acad. Sci. USA 99, 17025-17030 (2002).

5. Hernandez, M.E., Kappler, A. \& Newman, D.K. Phenazines and other redox-active antibiotics promote microbial mineral reduction. Appl. Environ. Microbiol. 70, 921-928 (2004).

6. Banin, E., Vasil, M.L. \& Greenberg, E.P. Iron and Pseudomonas aeruginosa biofilm formation. Proc. Natl. Acad. Sci. USA 102, 11076-11081 (2005).

7. Costerton, J.W., Lewandowski, Z., Caldwell, D.E., Korber, D.R. \& Lappinscott, H.M. Microbial biofilms. Annu. Rev. Microbiol. 49, 711-745 (1995).

8. Kolter, R., Siegele, D.A. \& Tormo, A. The stationary phase of the bacterial life cycle. Annu. Rev. Microbiol. 47, 855-874 (1993)

9. Laursen, J.B. \& Nielsen, J. Phenazine natural products: biosynthesis, synthetic analogues, and biological activity. Chem. Rev. 104, 1663-1686 (2004).

10. Kerr, J.R. Phenazine pigments: antibiotics and virulence factors. Infect. Dis. Rev. $\mathbf{2}$, 184-194 (2000).

11. Chin-A-Woeng, T.F.C. et al. Biocontrol by phenazine-1-carboxamide-producing Pseudomonas chlororaphis PCL1391 of tomato root rot caused by Fusarium oxysporum f. sp. radicis-lycopersici. Mol. Plant Microbe Interact. 11, 1069-1077 (1998).

12. Chin-A-Woeng, T.F.C., Bloemberg, G.V. \& Lugtenberg, B.J.J. Phenazines and their role in biocontrol by Pseudomonas bacteria. New Phytol. 157, 503-523 (2003).

13. O'Malley, Y.Q. et al. The Pseudomonas secretory product pyocyanin inhibits catalase activity in human lung epithelial cells. Am. J. Physiol. Lung Cell. Mol. Physiol. 285 L1077-L1086 (2003).

14. Lau, G.W., Hassett, D.J., Ran, H. \& Kong, F. The role of pyocyanin in Pseudomonas aeruginosa infection. Trends Mol. Med. 10, 599-606 (2004).

15. Look, D.C. et al. Pyocyanin and its precursor phenazine-1-carboxylic acid increase IL-8 and intercellular adhesion molecule-1 expression in human airway epithelial cells by oxidant-dependent mechanisms. J. Immunol. 175, 4017-4023 (2005).

16. Juhas, M., Eberl, L. \& Tummler, B. Quorum sensing: the power of cooperation in the world of Pseudomonas. Environ. Microbiol. 7, 459-471 (2005).

17. Hall-Stoodley, L. Bacterial biofilms: From the natural environment to infectious diseases. Nat. Rev. Microbiol. 2, 95-108 (2004).

18. Lazdunski, A.M., Ventre, I. \& Sturgis, J.N. Regulatory circuits and communication in Gram-negative bacteria. Nat. Rev. Microbiol. 2, 581-592 (2004).

19. Armstrong A.V. \& Stewart-Tull, D.E.S. The site of activity of extracellular products of Pseudomonas aeruginosa in the electron-transport chain in mammalian cell respiration. J. Med. Microbiol. 4, 263-270 (1971).

20. Ritz, D. \& Beckwith, J. Roles of thiol-redox pathways in bacteria. Annu. Rev. Microbiol. 55, 21-48 (2001) 
21. Villavicencio, R.T. The history of blue pus. J. Am. Coll. Surg. 187, 212-216 (1998).

22. Turner, J.M. \& Messenger, A.J. Occurrence, biochemistry and physiology of phenazine pigment production. Adv. Microb. Physiol. 27, 211-275 (1986).

23. Pirnay, J.P. et al. Global Pseudomonas aeruginosa biodiversity as reflected in a Belgian river. Environ. Microbiol. 7, 969-980 (2005).

24. Mavrodi, D.V. et al. Functional analysis of genes for biosynthesis of pyocyanin and phenazine-1-carboxamide from Pseudomonas aeruginosa PAO1. J. Bacteriol. 183, 6454-6465 (2001).

25. Delaney, S.M., Mavrodi, D.V., Bonsall, R.F. \& Thomashow, L.S. phzO, a gene for biosynthesis of 2-hydroxylated phenazine compounds in Pseudomonas aureofaciens 30-84. J. Bacteriol. 183, 318-327 (2001).

26. Chin-A-Woeng, T.F.C., Thomas-Oates, J.E., Lugtenberg, B.J.J. \& Bloemberg, G.V. Introduction of the phzH gene of Pseudomonas chlororaphis PCL1391 extends the range of biocontrol ability of phenazine-1- carboxylic acid-producing Pseudomonas spp. strains. Mol. Plant Microbe Interact. 14, 1006-1015 (2001).

27. Beifuss, U. \& Tietze, M. Methanophenazine and other natural biologically active phenazines. Top. Curr. Chem. 244, 77-113 (2005).

28. Rao, Y.M. \& Sureshkumar, G.K. Oxidative-stress-induced production of pyocyanin by Xanthomonas campestris and its effect on the indicator target organism, Escherichia coli. J. Ind. Microbiol. Biotechnol. 25, 266-272 (2000).

29. Lau, G.W., Hassett, D.J. \& Britigan, B.E. Modulation of lung epithelial functions by Pseudomonas aeruginosa. Trends Microbiol. 13, 389-397 (2005).

30. Wilson, R. et al. Measurement of Pseudomonas aeruginosa phenazine pigments in sputum and assessment of their contribution to sputum sol toxicity for respiratory epithelium. Infect. Immun. 56, 2515-2517 (1988).

31. Lau, G.W., Ran, H., Kong, F., Hassett, D.J. \& Mavrodi, D. Pseudomonas aeruginosa pyocyanin is critical for lung infection in mice. Infect. Immun. 72, 4275-4278 (2004).

32. Finnan, S. Genome diversity of Pseudomonas aeruginosa isolates from cystic fibrosis patients and the hospital environment. J. Clin. Microbiol. 42, 5783-5792 (2004).

33. Lau, G.W., Ran, H., Kong, F., Hassett, D.J. \& Mavrodi, D. Pseudomonas aeruginosa pyocyanin is critical for lung infection in mice. Infect. Immun. 72, 4275-4278 (2004).

34. O'Malley, Y.Q., Reszka, K.J., Spitz, D.R., Denning, G.M. \& Britigan, B.E. Pseudomonas aeruginosa pyocyanin directly oxidizes glutathione and decreases its levels in airway epithelial cells. Am. J. Physiol. Lung Cell. Mol. Physiol. 287, L94-103 (2004).

35. Hassan, H.M. \& Fridovich, I. Mechanism of the antibiotic action pyocyanine. J. Bacteriol. 141, 156-163 (1980).

36. Britigan, B.E., Railsback, M.A. \& Cox, C.D. The Pseudomonas aeruginosa secretory product pyocyanin inactivates alpha(1) protease inhibitor: implications for the pathogenesis of cystic fibrosis lung disease. Infect. Immun. 67, 1207-1212 (1999).

37. Hassett, D.J., Charniga, L., Bean, K., Ohman, D.E. \& Cohen, M.S. Response of Pseudomonas aeruginosa to pyocyanin: mechanisms of resistance, antioxidant defenses, and demonstration of a manganese-cofactored superoxide dismutase. Infect. Immun. 60, 328-336 (1992).

38. Britigan, B.E. et al. Interaction of the Pseudomonas aeruginosa secretory products pyocyanin and pyochelin generates hydroxyl radical and causes synergistic damage to endothelial cells: implications for Pseudomonas associated tissue injury. J. Clin. Invest. 90, 2187-2196 (1992)

39. Hassan, H.M. \& Fridovich, I. Intracellular production of superoxide radical and of hydrogen-peroxide by redox active compounds. Arch. Biochem. Biophys. 196, 385-395 (1979).

40. Ohman, D.E. Utilization of human respiratory secretions by mucoid Pseudomonas aerugionsa of cystic fibrosis origin. Infect. Immun. 37, 662-669 (1982).

41. Palmer, K.L. Cystic fibrosis sputum supports growth and cues key aspects of Pseudomonas aeruginosa physiology. J. Bacteriol. 187, 5267-5277 (2005).

42. Baron, S.S. \& Rowe, J.J. Antibiotic action of pyocyanin. Antimicrob. Agents Chemother. 20, 814-820 (1981).

43. Mazzola, M., Cook, R.J., Thomashow, L.S., Weller, D.M. \& Pierson, L.S., III. Contribution of phenazine antibiotic biosynthesis to the ecological competence of fluorescent pseudomonads in soil habitats. Appl. Environ. Microbiol. 58, 2616-2624 (1992).

44. Bankhead, S.B., Landa, B.B., Lutton, E., Weller, D.M. \& Gardener, B.B.M. Minimal changes in rhizobacterial population structure following root colonization by wild type and transgenic biocontrol strains. FEMS Microbiol. Ecol. 49, 307-318 (2004).

45. Vandenende, C.S., Vlasschaert, M. \& Seah, S.Y.K. Functional characterization of an aminotransferase required for pyoverdine siderophore biosynthesis in Pseudomonas aeruginosa PA01. J. Bacteriol. 186, 5596-5602 (2004).

46. Dewick, P.M. The biosynthesis of shikimate metabolites. Nat. Prod. Rep. 1, 451-469 (1984).

47. McDonald, M., Mavrodi, D.V., Thomashow, L.S. \& Floss, H.G. Phenazine biosynthesis in Pseudomonas fluorescens: branchpoint from the primary shikimate biosynthetic pathway and role of phenazine-1,6-dicarboxylic acid. J. Am. Chem. Soc. 123, 9459-9460 (2001).

48. Mavrodi, D.V. et al. A seven-gene locus for synthesis of phenazine-1-carboxylic acid by Pseudomonas fluorescens 2-79. J. Bacteriol. 180, 2541-2548 (1998).

49. Whiteley, M., Lee, K.M. \& Greenberg, E.P. Identification of genes controlled by quorum sensing in Pseudomonas aeruginosa. Proc. Natl. Acad. Sci. USA 96, 13904-13909 (1999).

50. Deziel, E. et al. Analysis of Pseudomonas aeruginosa 4-hydroxy-2-alkylquinolines (HAQs) reveals a role for 4-hydroxy-2-heptylquinoline in cell-to-cell communication. Proc. Natl. Acad. Sci. USA 101, 1339-1344 (2004).

51. Pierson, L.S., III, Keppenne, V.D. \& Wood, D.W. Phenazine antibiotic biosynthesis in Pseudomonas aureofaciens 30-84 is regulated by PhzR in response to cell density. J. Bacteriol. 176, 3966-3974 (1994).
52. Chin-A-Woeng, T.F. et al. Phenazine-1-carboxamide production in the biocontrol strain Pseudomonas chlororaphis PCL1391 is regulated by multiple factors secreted into the growth medium. Mol. Plant Microbe Interact. 14, 969-979 (2001).

53. Khan, S.R. et al. Activation of the phz operon of Pseudomonas fluorescens 2-79 requires the LuxR homolog PhzR, $\mathrm{N}$-(3-OH-hexanoyl)-L-homoserine lactone produced by the Luxl homolog Phzl, and a cis-acting phz box. J. Bacteriol. 187, 6517-6527 (2005).

54. van Rij, E.T., Wesselink, M., Chin, A.W.T.F., Bloemberg, G.V. \& Lugtenberg, B.J. Influence of environmental conditions on the production of phenazine-1-carboxamide by Pseudomonas chlororaphis PCL1391. Mol. Plant Microbe Interact. 17, 557-566 (2004).

55. Kim, E.J., Wang, W., Deckwer, W.D. \& Zeng, A.P. Expression of the quorum-sensing regulatory protein LasR is strongly affected by iron and oxygen concentrations in cultures of Pseudomonas aeruginosa irrespective of cell density. Microbiology 151, 1127-1138 (2005)

56. Chancey, S.T. Two-component transcriptional regulation of $\mathrm{N}$-acyl-homoserine lactone production in Pseudomonas aureofaciens. Appl. Environ. Microbiol. 65, 2294-2299 (1999).

57. Chancey, S.T., Wood, D.W., Pierson, E.A. \& Pierson, L.S., III. Survival of GacS/GacA mutants of the biological control bacterium Pseudomonas aureofaciens 30-84 in the wheat rhizosphere. Appl. Environ. Microbiol. 68, 3308-3314 (2002).

58. Whistler, C.A. \& Pierson, L.S., III. Repression of phenazine antibiotic production in Pseudomonas aureofaciens strain 30-84 by RpeA. J. Bacteriol. 185, 3718-3725 (2003).

59. Chin-A-Woeng. T.F., van den Broek, D., Lugtenberg, B.J. \& Bloemberg, G.V. The Pseudomonas chlororaphis PCL1391 sigma regulator psrA represses the production of the antifungal metabolite phenazine-1-carboxamide. Mol. Plant Microbe Interact. 18, 244-253 (2005).

60. Cases, I. Promoters in the environment: transcriptional regulation in its natural context. Nat. Rev. Microbiol. 3, 105-118 (2005).

61. Hooi, D.S.W. Differential immune modulatory activity of Pseudomonas aeruginosa quorum-sensing signal molecules. Infect. Immun. 72, 6463-6470 (2004).

62. Pesci, E.C. et al. Quinolone signaling in the cell-to-cell communication system of Pseudomonas aeruginosa. Proc. Natl. Acad. Sci. USA 96, 11229-11234 (1999).

63. Kaufmann, G.F. Revisiting quorum sensing: discovery of additional chemical and biological functions for 3-oxo- $N$-acylhomoserine lactones. Proc. Natl. Acad. Sci. USA 102 309-314 (2005).

64. Oliphant, C.M. Quinolones: a comprehensive review. Am. Fam. Physician 65, 455-464 (2002).

65. Friedheim, E. \& Michaelis, L. Potentiometric study of pyocyanine. J. Biol. Chem. 91 355-368 (1931).

66. Hernandez, M.E. \& Newman, D.K. Extracellular electron transfer. Cell. Mol. Life Sci. 58, 1562-1571 (2001).

67. Friedheim, E.A.H. Pyocyanine, an accessory respiratory pigment. J. Exp. Med. 54, 207-221 (1931).

68. Stewart-Tull, D.E.S. \& Armstrong, A.V. The effect of 1-hydroxyphenazine and pyocyanin from Pseudomonas aeruginosa on mammalian cell respiration. J. Med. Microbiol. 5, 67-73 (1972).

69. Muller, M. Scavenging of neutrophil-derived superoxide anion by 1-hydroxyphenazine a phenazine derivative associated with chronic Pseudomonas aeruginosa infection relevance to cystic fibrosis. Biochim. Biophys. Acta 1272, 185-189 (1995).

70. Learoyd, S.A., Kroll, R.G. \& Thurston, C.F. An investigation of dye reduction by foodborne bacteria. J. Appl. Bacteriol. 72, 479-485 (1992).

71. McKinlay, J.B. \& Zeikus, J.G. Extracellular iron reduction is mediated in part by neutral red and hydrogenase in Escherichia coli. Appl. Environ. Microbiol. 70, 3467-3474 (2004).

72. Deppenmeier, U. The membrane-bound electron transport system of Methanosarcina species. J. Bioenerg. Biomembr. 36, 55-64 (2004).

73. Beifuss, U., Tietze, M., Baumer, S. \& U., D. Methanophenazine: structure, total synthesis, and function of a new cofactor from methanogenic Archaea. Angew. Chem. Int Ed. Engl. 39, 2470-2473 (2000).

74. Deppenmeier, U. The unique biochemistry of methanogenesis. Prog. Nucleic Acid Res Mol. Biol. 71, 223-283 (2002).

75. Abken, H.J. et al. Isolation and characterization of methanophenazine and function of phenazines in membrane-bound electron transport of Methanosarcina mazei Gol. J. Bacteriol. 180, 2027-2032 (1998).

76. Fu, Y.C., Zhang, T.C. \& Bishop, P.L. Determination of effective oxygen diffusivity in biofilms grown in a completely mixed biodrum reactor. Water Sci. Tech. 29, 455-462 (1994).

77. Stewart, P.S. Diffusion in biofilms. J. Bacteriol. 185, 1485-1491 (2003).

78. de Graef, M.R., Alexeeva, S., Snoep, J.L. \& Teixeira de Mattos, M.J. The steady-state internal redox state (NADH/NAD) reflects the external redox state and is correlated with catabolic adaptation in Escherichia coli. J. Bacteriol. 181, 2351-2357 (1999).

79. Fultz, M.L. \& Durst, R.A. Mediator compounds for the electrochemical study of biological redox systems: a compilation. Anal. Chim. Acta 140, 1-18 (1982).

80. Bond, D.R., Holmes, D.E., Tender, L.M. \& Lovley, D.R. Electrode-reducing microorganisms that harvest energy from marine sediments. Science 295, 483-485 (2002).

81. Kim, B.H. et al. Enrichment of microbial community generating electricity using a fuelcell-type electrochemical cell. Appl. Microbiol. Biotechnol. 63, 672-681 (2004).

82. Lies, D.P. et al. Shewanella oneidensis MR-1 uses overlapping pathways for iron reduction at a distance and by direct contact under conditions relevant for biofilms. Appl. Environ Microbiol. 71, 4414-4426 (2005). 


\section{REVIEW}

83. Rabaey, K., Boon, N., Siciliano, S.D., Verhaege, M. \& Verstraete, W. Biofuel cells select for microbial consortia that self-mediate electron transfer. Appl. Environ. Microbiol. 70, 5373-5382 (2004).

84. Rabaey, K., Boon, N., Hofte, M. \& Verstraete, W. Microbial phenazine production enhances electron transfer in biofuel cells. Environ. Sci. Tech. 39, 3401-3408 (2005).

85. Cox, C.D. Role of pyocyanin in the acquisition of iron from transferrin. Infect. Immun. 52, 263-270 (1986).

86. King, E.O. Two simple media for the demonstration of pyocyanin and fluorescin. J. Lab. Clin. Med. 44, 301-307 (1954).

87. Fux, C.A. Survival strategies of infectious biofilms. Trends Microbiol. 13, 34-40 (2005).

88. Waite, R.D. Transcriptome analysis of Pseudomonas aeruginosa growth: comparison of gene expression in planktonic cultures and developing and mature biofilms. J. Bacteriol. 187, 6571-6576 (2005).

89. Handelsman, J. \& Wackett, L.P. Ecology and industrial microbiology: microbial diversity-sustaining the Earth and industry. Curr. Opin. Microbiol. 5, 237-239 (2002).
90. Clark, W.M. Azines. in Oxidation-Reduction Potentials of Organic Systems 412-419 (Williams \& Wilkins Company, Baltimore, 1960).

91. Meylan, W.M. \& Howard, P.H. Atom/fragment contribution method for estimating octanolwater partition coefficients. J. Pharm. Sci. 84, 83-92 (1995).

92. Mann, S. [Melanin-forming strains of Pseudomonas aeruginosa.] Arch. Mikrobiol. 65 359-379 (1969).

93. Byng, G.S., Eustice, D.C. \& Jensen, R.A. Biosynthesis of phenazine pigments in mutant and wild-type cultures of Pseudomonas aeruginosa. J. Bacteriol. 138, 846-852 (1979).

94. Wade, D.S. et al. Regulation of Pseudomonas quinolone signal synthesis in Pseudomonas aeruginosa. J. Bacteriol. 187, 4372-4380 (2005).

95. White, D. The Physiology and Biochemistry of Prokaryotes (Oxford University Press, New York, 2000).

96. Williamson, N.R. et al. Biosynthesis of the red antibiotic, prodigiosin, in Serratia: identification of a novel 2-methyl-3-n-amyl-pyrrole (MAP) assembly pathway, definition of the terminal condensing enzyme, and implications for undecylprodigiosin biosynthesis in Streptomyces. Mol. Microbiol. 56, 971-989 (2005). 